\title{
Field quality in low- $\beta$ superconducting quadrupoles and impact on the beam dynamics for the Large Hadron Collider upgrade
}

\author{
Boris Bellesia, Jean-Pierre Koutchouk, and Ezio Todesco \\ Accelerator Technology Department, CERN, CH-1211 Geneva
}

(Received 12 February 2007; published 1 June 2007)

\begin{abstract}
A possible scenario for the luminosity upgrade of the Large Hadron Collider is based on large aperture quadrupoles to lower $\beta^{*}$ in the interaction regions. Here we analyze the measurements relative to the field quality of the RHIC and LHC superconducting quadrupoles to find out the dependence of field errors on the size of the magnet aperture. Data are interpreted in the framework of a Monte Carlo analysis giving the reproducibility in the coil positioning reached in each production. We show that this precision is likely to be independent of the magnet aperture. Using this result, we can carry out an estimate of the impact of the field quality on the beam dynamics for the collision optics.
\end{abstract}

DOI: 10.1103/PhysRevSTAB.10.062401

PACS numbers: 84.71.Ba, 41.85.Gy, 29.27.-a

\section{INTRODUCTION}

The low- $\beta$ insertion in the interaction region of the Large Hadron Collider (LHC) consists of a triplet of superconducting quadrupoles [1-3] to focus the beam in the interaction point (IP). In the present baseline, the quadrupole aperture allows reaching a $\beta^{*}$ value of $0.55 \mathrm{~m}$ in the IP. In these quadrupoles, the $\beta$ functions at collision reach $4400 \mathrm{~m}$, corresponding to a beam size radius at $10 \sigma$ of $\sim 15 \mathrm{~mm}$, and requiring a quadrupole aperture of $70 \mathrm{~mm}$ [1].

Studies about how to improve the LHC luminosity have been started since 2002 [4-8]; a possible way is to decrease $\beta^{*}$ in the IP to values of $0.25 \mathrm{~m}$ or even less, thus requiring larger apertures in the triplet. For instance, $90 \mathrm{~mm}$ aperture $\mathrm{Nb}_{3} \mathrm{Sn}$ magnets are being built in the framework of the LHC Accelerator Research Program [9]. Recent studies [10-13] analyzed layouts with even larger apertures. A large aperture is used not only for allowing a larger $\beta$ function in the triplet, giving a smaller $\beta^{*}$ in the IP, but it can be used to have an additional shielding to prevent power deposition in the magnet coils. Moreover, a larger aperture allows increasing the collimator gap for a given collimator efficiency, thus reducing the impedance that presently is one of the limits to reaching nominal intensity in the LHC beam [14].

The natural questions that arise are if the geometric aberrations in the presence of large beta functions in the quadrupoles can become critical, and if they can be corrected. In this paper we use the data relative to the productions of the LHC and of the Relativistic Heavy Ion Collider [15] (RHIC) magnets to work out the dependence of the field errors on the magnet aperture, following the approaches outlined in [16-20]. We then estimate how the obtained scaling for the field errors affects typical parameters of the beam dynamics in collision optics, where the motion is dominated by the field imperfections of the low- $\beta$ quadrupoles.
In Sec. II, we present the field quality obtained in seven productions of superconducting quadrupoles, and we associate to these data an estimate of the reproducibility in positioning the coil blocks using a Monte Carlo method. In Sec. III we propose a scaling law for the field errors on the magnet aperture, and a numerical check is carried out on realistic coil layouts with apertures ranging from 50 to $200 \mathrm{~mm}$. In Sec. IV the impact on the beam dynamics is evaluated. Conclusions are given in Sec. V.

\section{FIELD QUALITY IN RHIC AND LHC QUADRUPOLES}

We analyze the data relative to 7 types of $\mathrm{Nb}$-Ti quadrupoles that have been built for the RHIC and for the LHC projects. Apertures (defined as bore diameter), operational gradients and peak fields, temperatures, and magnetic lengths are listed in Table I.

The magnetic field in a quadrupole is expressed as a multipolar expansion

$$
B_{y}+i B_{x}=10^{-4} B_{2} \sum_{n=2}^{\infty}\left(b_{n}+i a_{n}\right)\left(\frac{x+i y}{R_{\mathrm{ref}}}\right)^{n-1},
$$

where $b_{2}=10^{4}$ by definition, $B_{2}$ is the main component in $T$, and $R_{\text {ref }}$ is a reference radius, usually set as one-third of the aperture diameter, to have dimensionless multipoles $\left(b_{n}, a_{n}\right)$. Since the multipoles are also normalized by an additional factor $10^{-4}$, they are said to be expressed in units, where 1 unit is $10^{-4}$ (e.g., $0.01 \%$, or $100 \mathrm{ppm}$ ) times the main component. The measured standard deviations of $\left(b_{n}, a_{n}\right)$ are listed in Table II. To ease the comparison, multipoles have been all normalized to a reference radius equal to $1 / 3$ of the aperture diameter. The RHIC quadrupoles Q1-Q3 have the same cross section, but different lengths (see Table I), and therefore are expected to have similar standard deviation of measured multipoles, as observed. The same holds for the LHC MQM, MQMC, and MQML. 
TABLE I. Features of superconducting quadrupoles in RHIC and LHC.

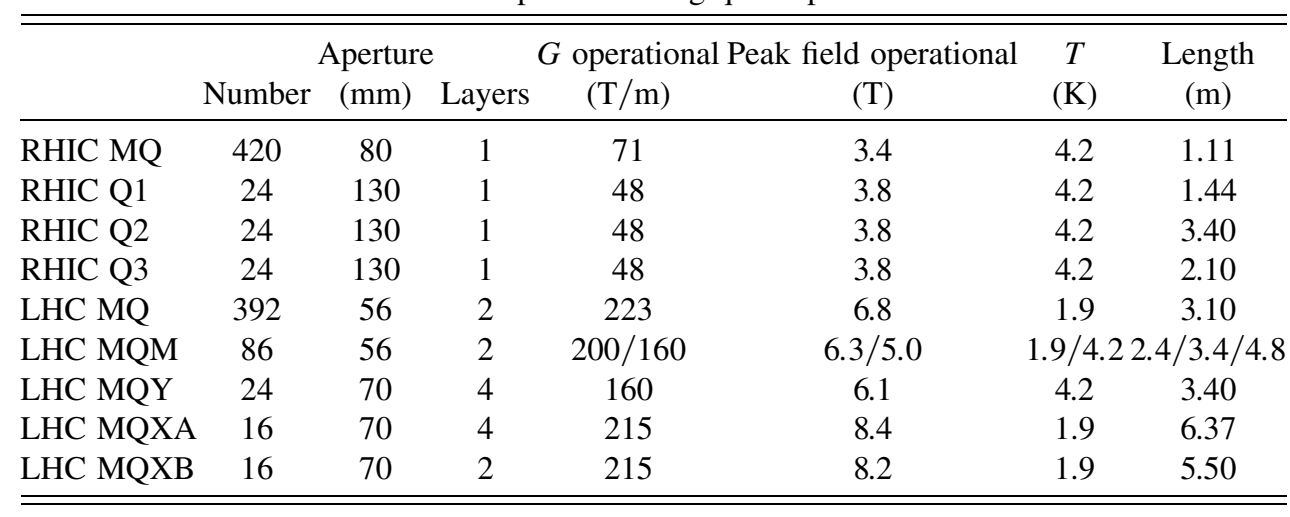

TABLE II. Standard deviation of measured multipoles in RHIC and LHC quadrupoles, reference radius taken as $2 / 3$ of aperture.

\begin{tabular}{lccccccccccc}
\hline \hline & Ap $(\mathrm{mm})$ & $\mathrm{T}(\mathrm{K})$ & Measured & $b_{3}$ & $b_{4}$ & $b_{5}$ & $b_{6}$ & $a_{3}$ & $a_{4}$ & $a_{5}$ & $a_{6}$ \\
\hline RHIC MQ & 80 & 300 & 380 & 1.69 & 1.02 & 0.54 & 0.48 & 1.75 & 1.02 & 0.53 & 0.33 \\
RHIC MQ & 80 & 4.2 & 91 & 1.86 & 1.50 & 1.74 & 0.70 & 1.77 & 0.97 & 1.55 & 0.35 \\
RHIC Q1 & 130 & 4.2 & 26 & 0.52 & 0.56 & 0.34 & 0.88 & 0.66 & 0.32 & 0.40 & 0.18 \\
RHIC Q2 & 130 & 4.2 & 27 & 0.50 & 0.24 & 0.30 & 0.57 & 0.60 & 0.28 & 0.39 & 0.40 \\
RHIC Q3 & 130 & 4.2 & 13 & 0.86 & 0.65 & 0.32 & 0.19 & 0.69 & 0.42 & 0.21 & 0.10 \\
LHC MQ & 56 & 300 & 402 & 1.81 & 0.42 & 0.70 & 1.36 & 2.22 & 2.29 & 0.73 & 0.48 \\
LHC MQ & 56 & 1.9 & 39 & 1.65 & 0.38 & 0.61 & 1.82 & 2.05 & 1.80 & 0.71 & 0.45 \\
LHC MQM & 56 & 300 & 46 & 1.83 & 0.97 & 0.65 & 1.01 & 2.07 & 1.13 & 0.57 & 0.35 \\
LHC MQMC & 56 & 300 & 14 & 1.39 & 0.58 & 0.63 & 1.09 & 1.63 & 1.14 & 0.63 & 0.40 \\
LHC MQML & 56 & 300 & 38 & 1.64 & 0.57 & 0.64 & 0.88 & 1.63 & 0.99 & 0.42 & 0.29 \\
LHC MQY & 70 & 4.2 & 11 & 1.39 & 0.49 & 0.39 & 0.58 & 1.28 & 0.80 & 0.57 & 0.24 \\
LHC MQXA & 70 & 1.9 & 19 & 0.60 & 0.28 & 0.13 & 0.42 & 0.75 & 0.70 & 0.15 & 0.11 \\
LHC MQXB & 70 & 1.9 & 8 & 0.73 & 0.24 & 0.42 & 1.03 & 1.08 & 0.92 & 0.33 & 0.70 \\
\hline \hline
\end{tabular}

For large productions (more than 100 magnets, i.e., RHIC MQ and LHC MQ) we give both the room temperature measurements, carried out on all the magnets, and the $1.9 / 4.2 \mathrm{~K}$ measurements in collision, carried out on a limited sample (25\% for RHIC, $10 \%$ for LHC). This allows on the one hand to cross-check the data, and on the other hand to verify if the geometric component measured at room temperature is dominant over the other effects present at collision energy, namely, the warm-cold correlation, the iron saturation, and electromagnetic force deformations. Data of Table II show that the rms multipoles measured at room temperature and in operational conditions are rather similar, especially in the LHC case. For the other magnets, we present measurements at 1.9/4.2 K, with the exception of the LHC MQM-type series, where room temperature measurements are given.

The data confirm that the spread of the multipoles is mainly due to the geometric components, i.e., to the reproducibility in the cable positioning in the magnet aperture. Please note that this reproducibility is not related to the absolute precision in positioning the coil with respect to the design: we always assume that after a few iterations in the coil design one manages to obtain the target values for the field harmonics.

Using the approach outlined in [19], we postprocessed the spread of the measured multipoles to evaluate the standard deviation of the reproducibility in the positioning of the coil blocks. For each coil layout we carry out a simulation where each cable block is randomly moved with an rms amplitude of $d_{s}=0.1 \mathrm{~mm}$, and the corresponding spread in the multipoles $\sigma_{b n}^{s}, \sigma^{s}$ an are calculated. The multipole spread is linear in $d_{s}$ over the range of interest $(0.01$ to $0.10 \mathrm{~mm})$. We define the discrepancy between the simulated values with an rms amplitude $d$ and the measured values $\left(\sigma_{b n}^{m}, \sigma_{a n}^{m}\right)$ as

$$
\begin{aligned}
\chi^{2}(d)= & \frac{1}{2(N-2)} \sum_{n=3}^{N}\left\{\left[\log \left(\frac{d}{\sigma_{b n}^{m}} \frac{\sigma_{b n}^{s}}{d_{s}}\right)\right]^{2}\right. \\
& \left.+\left[\log \left(\frac{d}{\sigma_{a n}^{m}} \frac{\sigma_{a n}^{s}}{d_{s}}\right)\right]^{2}\right\} .
\end{aligned}
$$

Minimizing the discrepancy with respect to $d$, we obtain the standard deviation $d_{0}$ of the reproducibility of coil positioning that best fits the measured multipole spread 


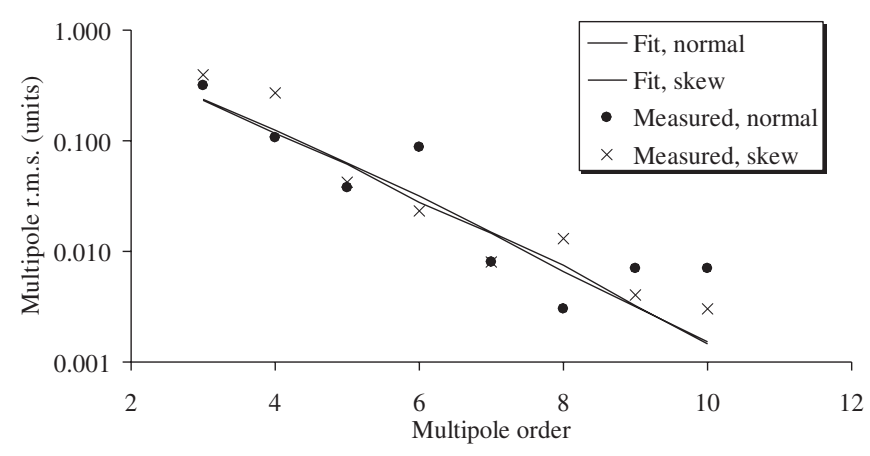

FIG. 1. Measured rms of multipole in LHC MQXA at $1.9 \mathrm{~K}$ (markers), and best fit through simulations with $d_{0}=0.011 \mathrm{~mm}$ (lines).

$$
d_{0}=d_{s} \exp \left\{\frac{1}{2(N-2)} \sum_{n=3}^{N}\left[\log \left(\frac{\sigma_{b n}^{m}}{\sigma_{b n}^{s}}\right)+\log \left(\frac{\sigma_{a n}^{m}}{\sigma_{a n}^{s}}\right)\right]\right\}
$$

and we define the error of the fit as

$$
\varepsilon=\frac{1}{N-2} \sum_{n=3}^{N}\left\{\left|1-\frac{d_{0} \sigma_{b n}^{s}}{d_{s} \sigma_{b n}^{m}}\right|+\left|1-\frac{d_{0} \sigma_{a n}^{s}}{d_{s} \sigma_{a n}^{m}}\right|\right\}
$$

Data given in Table II have been analyzed using this approach, and the reproducibility $d_{0}$ with the average relative error have been worked out (see Figs. 1 and 2), with $N=8$.

One finds out that $d_{0}$ ranges from 0.010 to $0.030 \mathrm{~mm}$, without a clear dependence on the aperture: quadrupoles for $56 \mathrm{~mm}$ have a positioning of $0.020 \mathrm{~mm}$ (LHC MQM types) to $0.030 \mathrm{~mm}$ (LHC MQ types). $70 \mathrm{~mm}$ aperture quadrupoles have a better $d_{0} \sim 0.010$ to $0.025 \mathrm{~mm}$, RHIC 80 and $130 \mathrm{~mm}$ apertures have $d_{0} \sim 0.015 \mathrm{~mm}$. One could argue that the large reproducibility for the LHC MQ $(0.030 \mathrm{~mm})$ is due to the presence of five different cable manufacturers. However, splitting the data according to the cable manufacturer does not reduce the observed tolerance.

The average error associated to this analysis is always rather large, i.e., $20 \%$ to $60 \%$. Nevertheless, one can draw the following guidelines: (i) The best hypothesis that can

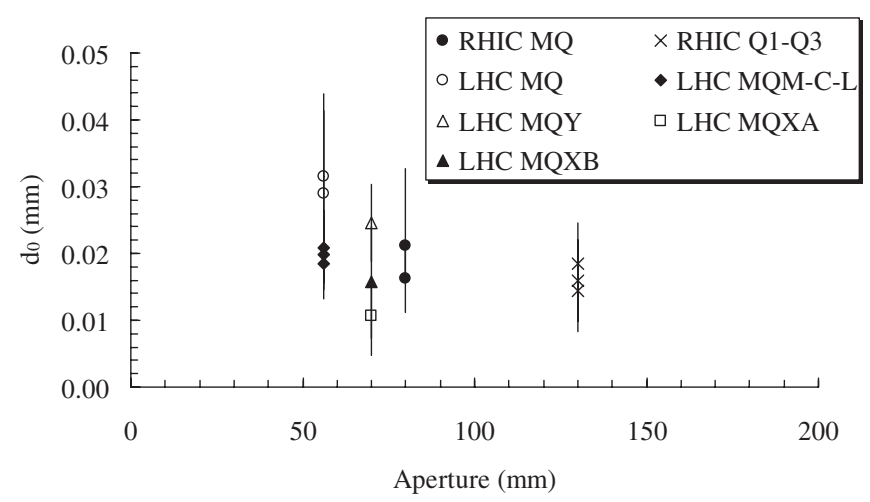

FIG. 2. Coil positioning versus aperture derived from magnetic measurements of 7 types of superconducting quadrupoles.

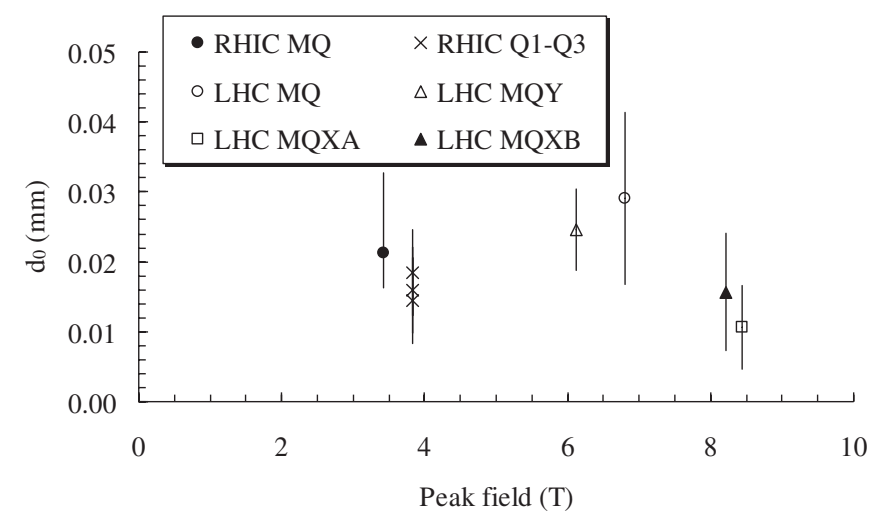

FIG. 3. Coil positioning versus peak field derived from magnetic measurements of 6 types of superconducting quadrupoles.

be done is that the reproducibility of coil positioning is independent of the aperture, $\sim 0.015-0.020 \mathrm{~mm}$, with an indeterminacy of about $\pm 40 \%$. (ii) The hypothesis that $d_{0}$ would linearly scale with the aperture should therefore be rejected. (iii) No dependence is found on the peak field (see Fig. 3), thus excluding that Lorentz forces play a relevant role in the reproducibility of the coil position in operational conditions.

\section{A SCALING LAW FOR FIELD ERRORS}

Let us consider a quadrupole of aperture $\phi$, coil thickness $w$, characterized by a set of random field components $\sigma$ generated by a random movement of coil blocks with rms $d$. We set the reference radius $R_{\text {ref }}$ as $1 / 3$ of the aperture diameter. The multipoles defined in (1) are invariant under a rescaling of the coordinates and of the reference radius. Therefore, if we increase the aperture by a factor $\alpha$, and consequently multiply the coil thickness, the reference radius, and the block movement by the same factor (see Fig. 4), the random field components would be invariant:

$$
\sigma\left(b_{n}, a_{n} ; \alpha \phi, \alpha d, \alpha R_{\text {ref }}\right)=\sigma\left(b_{n}, a_{n} ; \phi, d, R_{\text {ref }}\right) .
$$

However, the analysis of the data carried out in the pre-
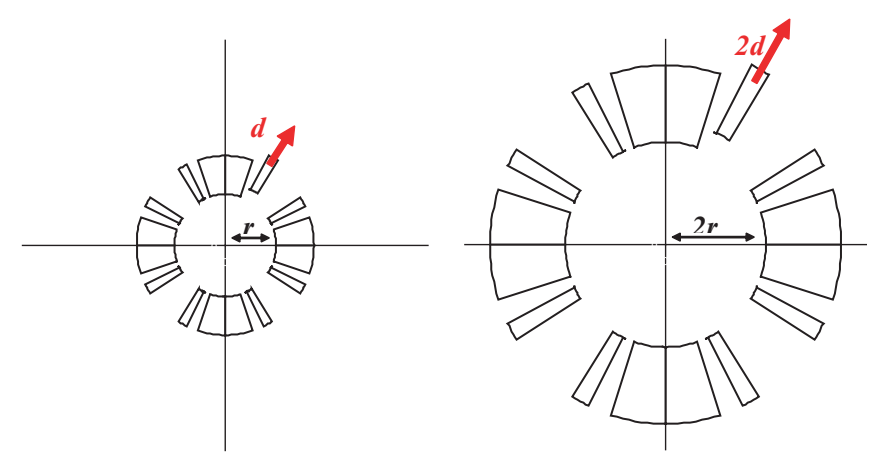

FIG. 4. (Color) Rescaling of a coil layout and of a block displacement by $\alpha=2$, leading to the same multipolar errors. 
TABLE III. Features of four coil layouts, and expected $b_{3}$ and $b_{4}$ rms based on a Monte Carlo with random movements of $0.015 \mathrm{~mm}$.

\begin{tabular}{ccccccc}
\hline \hline $\begin{array}{c}\text { Aperture } \\
(\mathrm{mm})\end{array}$ & $\begin{array}{c}\text { Coil } \\
\text { surface } \\
\left(\mathrm{mm}^{2}\right)\end{array}$ & $\begin{array}{c}\text { Equivalent } \\
\text { width } \\
(\mathrm{mm})\end{array}$ & $\begin{array}{c}\text { Gradient } \\
(\mathrm{T} / \mathrm{m})\end{array}$ & $\begin{array}{c}R_{\text {ref }} \\
(\mathrm{mm})\end{array}$ & $\begin{array}{c}\sigma\left(b_{3}\right) \\
(\text { units })\end{array}$ & $\begin{array}{c}\sigma\left(b_{4}\right) \\
(\text { units })\end{array}$ \\
\hline 50 & 4387 & 30.7 & 314 & 16.7 & 0.97 & 0.61 \\
100 & 12952 & 46.1 & 184 & 33.3 & 0.47 & 0.30 \\
150 & 26321 & 61.4 & 131 & 50.0 & 0.28 & 0.18 \\
200 & 38437 & 76.8 & 101 & 66.7 & 0.21 & 0.15 \\
\hline \hline
\end{tabular}

vious section shows that the reproducibility in coil positioning is likely to be independent of the coil aperture; therefore one has

$$
\sigma\left(b_{n}, a_{n} ; \alpha \phi, d, \alpha R_{\text {ref }}\right)=\frac{1}{\alpha} \sigma\left(b_{n}, a_{n} ; \phi, d, R_{\text {ref }}\right),
$$

i.e., the multipole spread scales with the inverse of the aperture radius.

This simple scaling is strictly valid for quadrupoles of identical cross sections (see Fig. 4), apart from a scale parameter. In practice, increasing the quadrupole aperture has a significant impact on the cross section. To verify that the scaling (6) remains true, we designed four realistic quadrupoles with apertures ranging from 25 to $100 \mathrm{~mm}$, and studied the random harmonics for a given $d$ with the Monte Carlo method. In each case, the same cable of $15.1 \mathrm{~mm}$ width used for the main LHC quadrupole has been chosen, and the number of turns has been selected to provide $95 \%$ of the maximum critical gradient according to

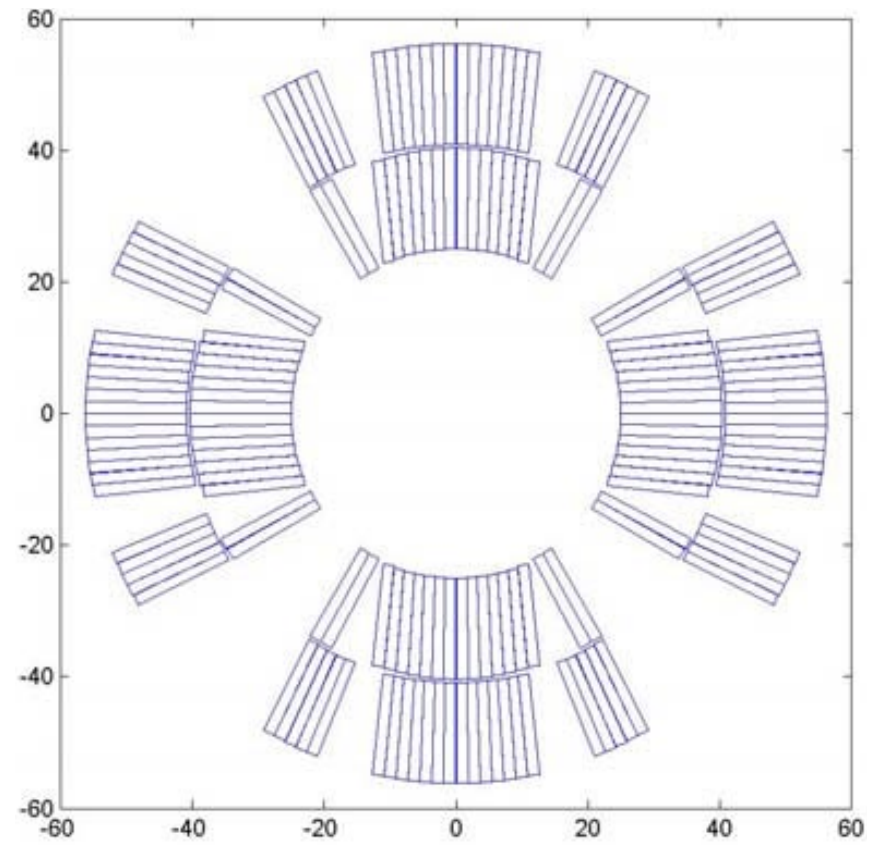

FIG. 5. (Color) Coil layout of a quadrupole with $50 \mathrm{~mm}$ aperture.

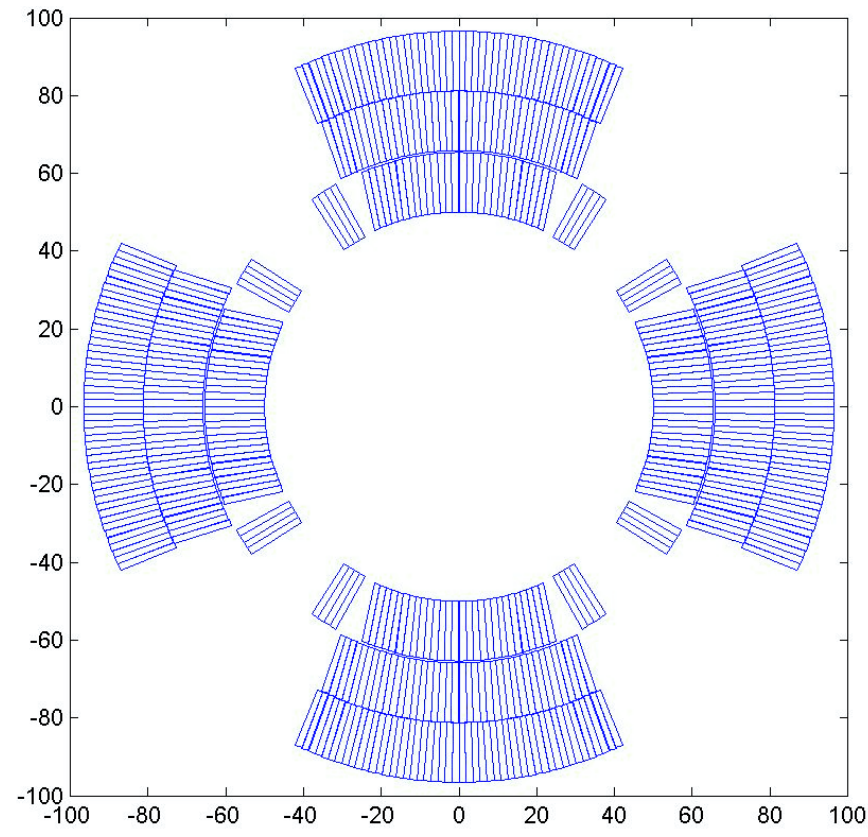

FIG. 6. (Color) Coil layout of a quadrupole with $100 \mathrm{~mm}$ aperture.

the scaling law presented in [21]. For each layout we provide (see Table III) the total coil area $A$, and the equivalent width of the coil, i.e., the width of the coil corresponding to a $30^{\circ}$ sector with the same area:

$$
w_{\text {eq }} \equiv\left(\sqrt{1+\frac{3 A}{2 \pi r^{2}}}-1\right) \frac{\phi}{2} .
$$

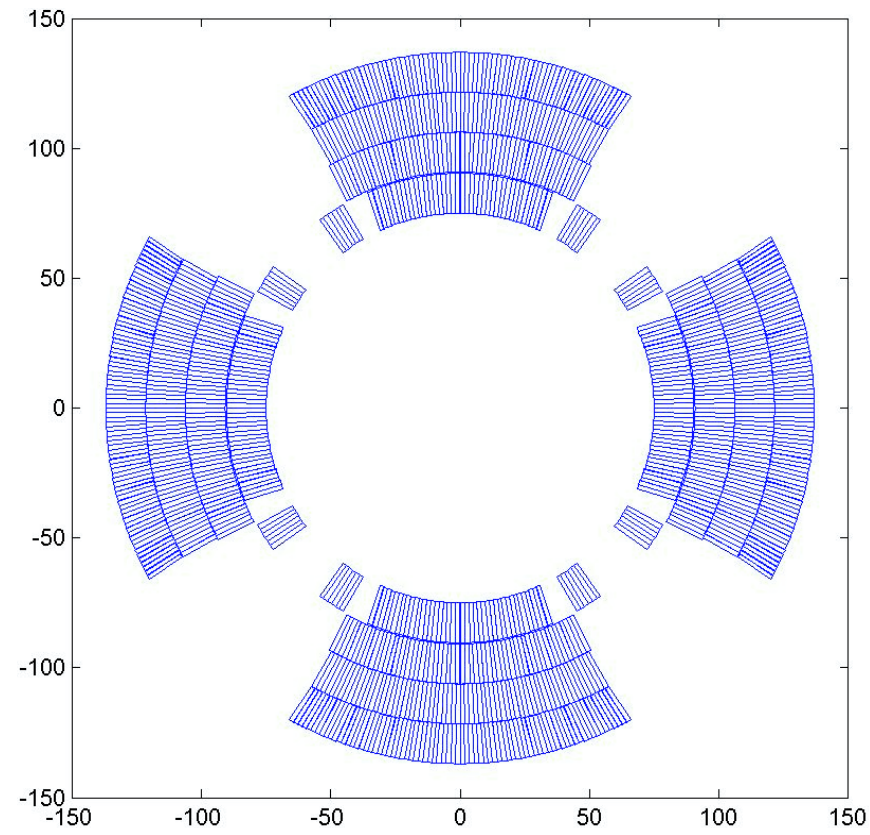

FIG. 7. (Color) Coil layout of a quadrupole with $150 \mathrm{~mm}$ aperture. 


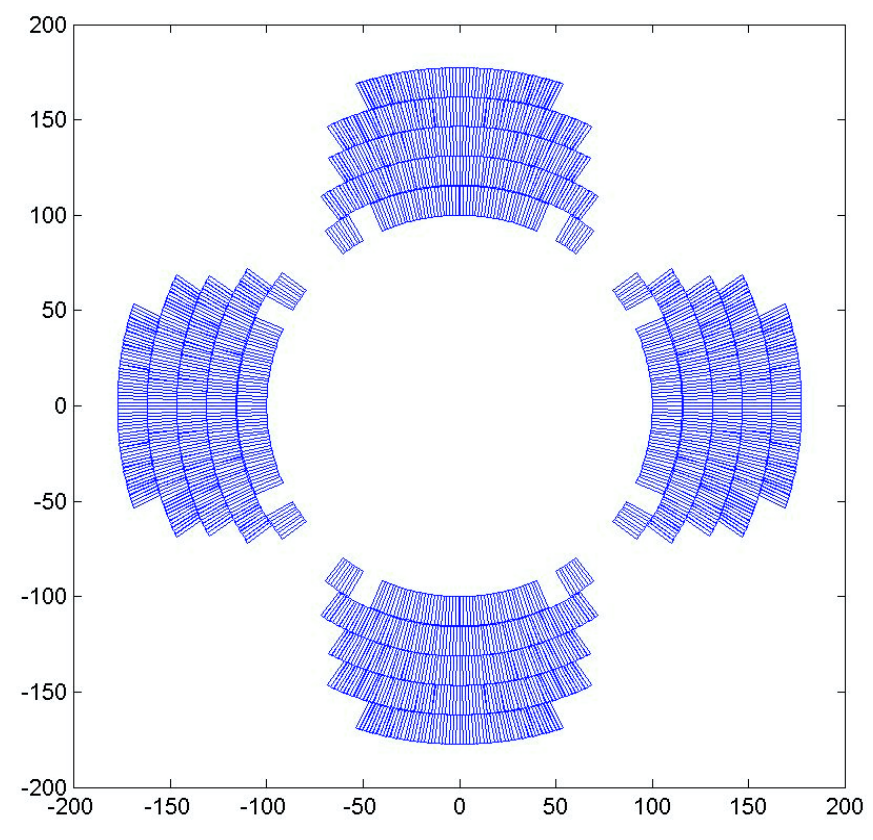

FIG. 8. (Color) Coil layout of a quadrupole with $200 \mathrm{~mm}$ aperture.

Field quality has been optimized to set $b_{6}$ and $b_{10}$ to zero within a fraction of unit, the inner layer being composed by two blocks, based on the layout of a $36^{\circ}$ sector coil with a wedge between $24^{\circ}$ and $30^{\circ}$ (see Figs. 5-8). For the large aperture cases, the outer blocks have little impact on $b_{10}$, and therefore a single block has been used. In all cases, a design without grading has been chosen. The quadrupole parameters, assuming a typical $\mathrm{Nb}$-Ti cable at $1.9 \mathrm{~K}$ with a filling factor of 0.25 , are given in Table III. In the last column, the rms of $b_{3}$ and $b_{4}$ are estimated for a random movement of coil blocks of rms $d=0.015 \mathrm{~mm}$. The multipole spread is proportional to the inverse of the aperture within 10\%; a similar behavior is found for the other multipoles (see Fig. 9). These simulations confirm that the scaling law proposed in (6) is valid for realistic coil

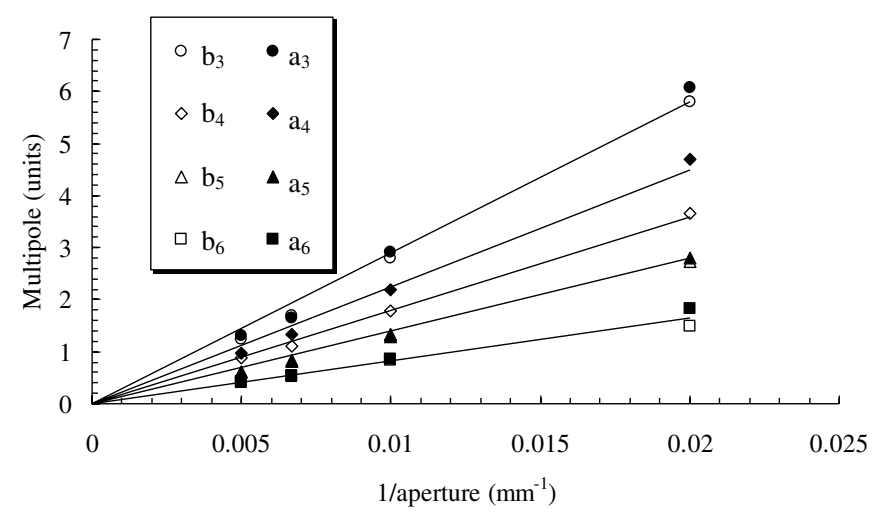

FIG. 9. Multipole rms obtained with 0.015 random movement versus inverse of the aperture. layouts, in the hypothesis that the reproducibility of coil positioning is independent of the aperture.

\section{IMPACT ON BEAM DYNAMICS}

We showed that large aperture quadrupoles are expected to have a better field quality; in this section we study the effect of this improvement on the beam dynamics in the collision optics, where, due to the high value of the $\beta$-functions, the contribution of low- $\beta$ quadrupoles is dominant.

\section{A. Detuning due to octupoles}

We first consider the 1st order amplitude-dependent tune shift induced by the octupolar term $b_{4}$ of an IR quadrupole, which is proportional to

$$
\Delta Q \propto \int K_{3} \beta^{2} d s
$$

where the normalized field derivative $K_{3}$ depends on the multipole [see Eq. (1)] according to

$$
K_{3} \equiv \frac{1}{B \rho} \frac{\partial^{3} B_{y}}{\partial x^{3}}=3 ! \frac{B_{2}}{B \rho} \frac{b_{4}}{R_{\mathrm{ref}}^{3}}=3 ! \frac{G}{B \rho} \frac{b_{4}}{R_{\mathrm{ref}}^{2}}
$$

and $G=B_{2} / R_{\text {ref }}$ is the nominal field gradient in $\mathrm{T} / \mathrm{m}$. If we increase the aperture by a factor $\alpha$

$$
\phi \rightarrow \bar{\phi}=\alpha \phi
$$

and the reference radius

$$
R_{\text {ref }} \rightarrow \bar{R}_{\text {ref }}=\alpha R_{\text {ref }}
$$

according to the results of the previous section, the multipoles rescale according to

$$
b_{4} \rightarrow \bar{b}_{4}=\frac{b_{4}}{\alpha} .
$$

The aperture $\phi$ required in a low- $\beta$ quadrupole is given by

$$
\phi=A+B \sqrt{\beta}
$$

where $\beta$ is the maximum beta function in the quadrupole, and $A$ and $B$ are constants, namely $A$ is related to mechanical tolerances, closed orbit, and shielding, and $B$ to the emittance. A sketch of the nominal LHC case, with the two beams within the beam pipe, is shown in Fig. 10. We now consider three alternative scenarios.

Scenario 1.-We assume that a larger aperture of the low- $\beta$ quadrupole is used to house a larger beam; in this case one has 


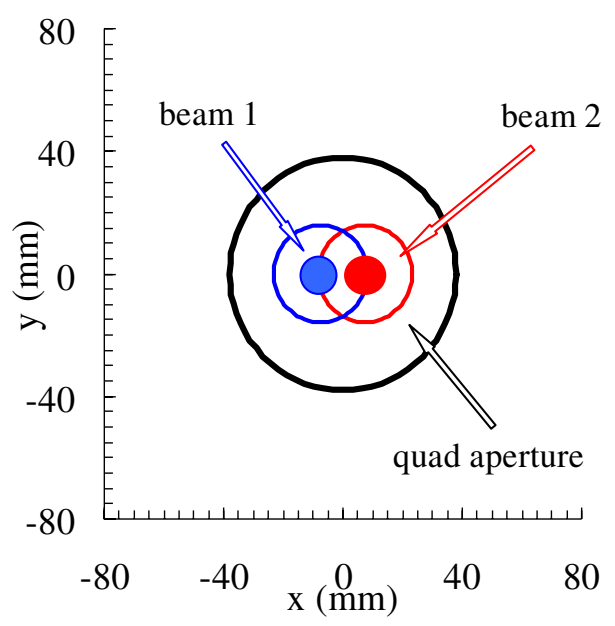

FIG. 10. (Color) Sketch of the beam location in the LHC IR quadrupole, nominal values $\left(\beta^{*}=55 \mathrm{~cm}\right)$.

$$
\beta=\left(\frac{\phi-A}{B}\right)^{2} \quad \bar{\beta}=\left(\frac{\bar{\phi}-A}{B}\right)^{2}=\left(\frac{\alpha \phi-A}{B}\right)^{2}
$$

and therefore

$$
\beta \rightarrow \bar{\beta}=\left(\frac{\alpha \phi-A}{\phi-A}\right)^{2} \beta=\alpha^{2}\left(\frac{\alpha \phi-A}{\alpha \phi-\alpha A}\right)^{2} \beta \equiv \alpha^{2} \eta^{2} \beta .
$$

For instance in the nominal layout of the LHC, one has $\phi=70 \mathrm{~mm}$ and $A=22 \mathrm{~mm}$, thus giving $\eta=1.2$ for doubling the aperture $\alpha=2$ (see Fig. 11). As a first order approximation, one can consider the integrated gradient as a constant,

$$
\int G d s \rightarrow \int G d s
$$

and therefore the integrated multipole scales with [see

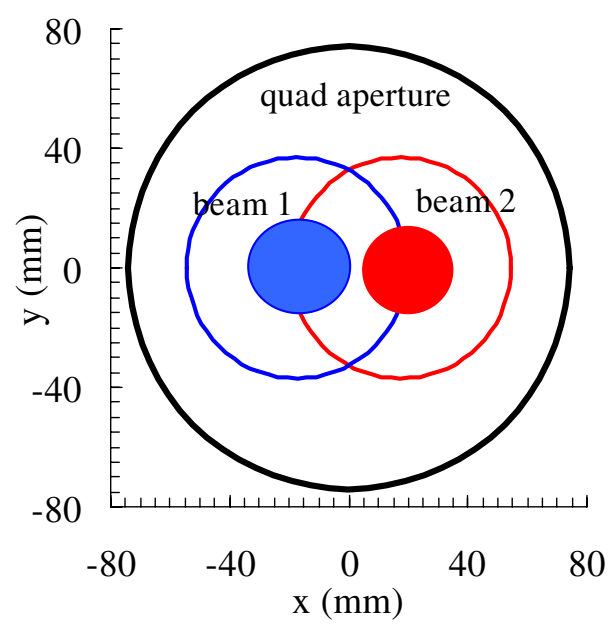

FIG. 11. (Color) Sketch of the beam location in the IR quadrupole, doubling the quadrupole aperture and reducing $\beta^{*}$ with respect to Fig. 10.

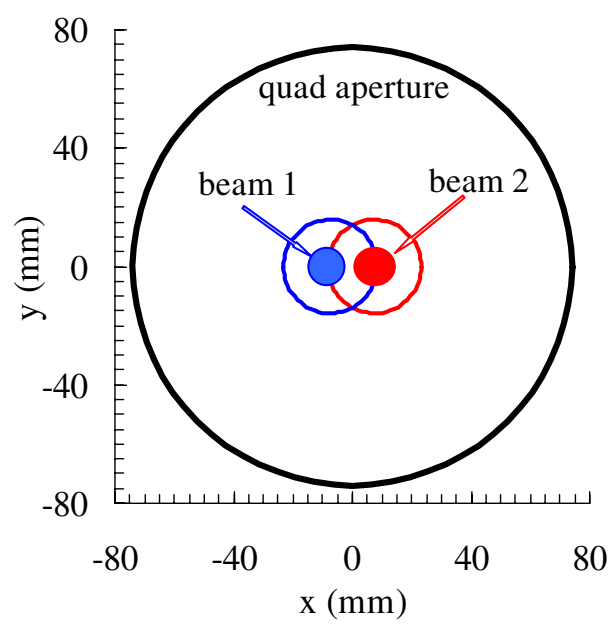

FIG. 12. (Color) Sketch of the beam location in the IR quadrupole, doubling the quadrupole aperture and keeping the same $\beta^{*}$ as in Fig. 10.

Eqs. (9), (11), and (12)]

$$
\begin{gathered}
\int K_{3} d s \rightarrow \alpha^{-3} \int K_{3} d s \\
\Delta Q \rightarrow \Delta \bar{Q}=\eta^{4} \alpha \Delta Q .
\end{gathered}
$$

In the case $\alpha=2$ (doubling the aperture of the triplet from 70 to $140 \mathrm{~mm}$ ), one obtains an increase of the detuning with amplitude by a factor $\alpha \eta^{4} \sim 4$.

Scenario 2.-We assume that the low- $\beta$ quadrupole aperture is increased to have an additional beam clearance, either for shielding or collimation (i.e., increasing $A$ in Eq. (13), see Fig. 12) but keeping the same beam size

$$
\beta \rightarrow \bar{\beta}=\beta
$$

and therefore the scaling is

$$
\Delta Q \rightarrow \Delta \bar{Q}=\alpha^{-3} \Delta Q .
$$

In the case of a double aperture $\alpha=2$ the detuning decreases by a factor 8 .

Scenario 3. - The increase by a factor $\alpha$ in the quadrupole aperture is associated to an increase $\delta$ in the beta function such that the detuning is invariant. Since

$$
\Delta Q \rightarrow \Delta \bar{Q}=\frac{\delta^{2}}{\alpha^{3}} \Delta Q
$$

the condition of invariance reads

$$
\alpha^{3}=\delta^{2} .
$$

For instance, doubling the aperture $\alpha=2$, one can still increase the $\beta$ function by a factor $\delta=2 \sqrt{2} \sim 2.8$ (compared to the factor 6 in scenario 1 ). 


\section{B. Aberrations proportional to field derivatives}

Let us consider a more general case, i.e., the dynamics in the transverse phase space induced by a multipolar kick in the triplet; we use the mapping approach [22]

$$
\left(\begin{array}{c}
x^{\prime} \\
p^{\prime}
\end{array}\right)=\mathbf{L}\left(p+\sum_{n=2}^{x} \frac{K_{n} \ell}{n !} x^{n}\right),
$$

where $\mathbf{L}$ is the one turn transfer matrix at the low- $\beta$ quadrupole, and

$$
K_{n} \ell \equiv \frac{1}{B \rho} \int \frac{\partial^{n} B_{y}}{\partial x^{n}} d s \sim \frac{1}{B \rho} \frac{b_{n+1}}{R_{\mathrm{ref}}{ }^{n-1}} \int G d s .
$$

Using (11) and (12), one finds that the scaling in the aperture induces the following scaling:

$$
K_{n} \ell \rightarrow \bar{K}_{n} \ell=\alpha^{-n} K_{n} \ell .
$$

We then rewrite the one turn map in terms of the CourantSnyder coordinates

$$
\left(\begin{array}{l}
\hat{x} \\
\hat{p}
\end{array}\right)=\left(\begin{array}{cc}
\frac{1}{\sqrt{\beta}} & 0 \\
-\frac{\alpha}{\sqrt{\beta}} & \sqrt{\beta}
\end{array}\right)\left(\begin{array}{l}
x \\
p
\end{array}\right),
$$

obtaining

$$
\left(\begin{array}{l}
\hat{x}^{\prime} \\
\hat{p}^{\prime}
\end{array}\right)=\mathbf{R}\left(\hat{p}+\sum_{n=2} \frac{K_{n} \ell}{n !} \beta^{(n+1) / 2} \hat{x}^{n}\right),
$$

where $\mathbf{R}$ is a rotation matrix. In the hypothesis (15), the nonlinear term scales as

$$
\left[\sum_{n=2} \frac{K_{n}}{n !} \beta^{(n+1) / 2} \hat{x}^{n}\right] \rightarrow \alpha \eta^{n+1}\left[\sum_{n=2} \frac{K_{n}}{n !} \beta^{(n+1) / 2} \hat{x}^{n}\right]
$$

whereas if the larger aperture is not used for the beam one has (19) and therefore

$$
\left[\sum_{n=2} \frac{K_{n}}{n !} \beta^{(n+1) / 2} \hat{x}^{n}\right] \rightarrow \alpha^{-n}\left[\sum_{n=2} \frac{K_{n}}{n !} \beta^{(n+1) / 2} \hat{x}^{n}\right] .
$$

If the quadrupole aperture is increased by $\alpha$ and the beta function by $\delta$, in order to keep the same nonlinear term of order $n$, we have to satisfy the conditions

$$
\alpha^{n}=\delta^{(n+1) / 2}
$$

thus giving

$$
\delta=\frac{1}{\alpha^{2 / n+1}} \alpha^{2} .
$$

For high orders the relation tends to $\delta=\alpha^{2}$, i.e., doubling the aperture $\alpha=2$ one can multiply the beta function by a factor $\delta=4$. The lowest order term with $n=2$ gives $\delta=$ $\alpha^{4 / 3}$ and therefore the term is preserved if a double aperture is associated to an increase of the $\beta$ function of a factor $\delta=2^{4 / 3} \sim 2.5$. One concludes that one can still increase the beta functions but keeping the same aberrations.

\section{Detuning induced by sextupoles}

To have a hint on what happens to the higher order aberrations, we consider the first order tune shift due to the second order in the sextupole

$$
\Delta Q \propto \iint\left(K_{2}\right)^{2} \beta^{3} d s_{1} d s_{2},
$$

where

$$
K_{2} \equiv \frac{1}{B \rho} \frac{\partial^{2} B_{y}}{\partial x^{2}}=2 ! \frac{B_{2}}{B \rho} \frac{b_{3}}{R_{\mathrm{ref}}{ }^{2}}=2 ! \frac{G}{B \rho} \frac{b_{3}}{R_{\mathrm{ref}}} .
$$

Using the same argument as before, one has

$$
\iint\left(K_{2}\right)^{2} d s_{1} d s_{2} \rightarrow \alpha^{-4} \iint\left(K_{2}\right)^{2} d s_{1} d s_{2}
$$

and for the scenario 1 , one has

$$
\Delta Q \rightarrow \Delta \bar{Q}=\eta^{6} \alpha^{2} \Delta Q,
$$

i.e. a doubling of the aperture increases the second order tune shift due to sextupoles of a factor $\sim 12$. Therefore, the increase of the higher order terms is more important with respect to the previous case. On the other hand, for the scenario 2, one finds a larger reduction

$$
\Delta Q \rightarrow \Delta \bar{Q}=\alpha^{-4} \Delta Q .
$$

i.e. a factor 16 improvement for a double aperture. A rescaling of the aperture by $\alpha$ and of the beta function by $\delta$ gives

$$
\Delta Q \rightarrow \Delta \bar{Q}=\alpha^{-4} \delta^{3} \Delta Q,
$$

i.e. if $\alpha=2$ and $\delta=2^{4 / 3}$ as in the previous scenario 3, also the second order is preserved.

Summarizing, an increase of the quadrupole aperture which is totally used to increase the beam size (scenario 1) produces significantly higher aberrations. On the other hand, if the larger aperture is associated to the same beam size (scenario 2), and is used for additional shielding or for improving collimation, the resulting aberrations are significantly lower. An increase of the beam size that does not fully exploit the larger aperture but keeping the same geometric aberrations can be worked out (scenario 3).

\section{CONCLUSIONS}

A scheme for the upgrade on the LHC low- $\beta$ insertions is based on large aperture superconducting quadrupoles: in this work we aimed at finding scaling laws for evaluating the expected field quality and the impact on the beam dynamics in the collision optics. We analyzed data relative to the production of the LHC and RHIC quadrupoles, showing how to derive from the magnetic measurements the reproducibility of coil positioning reached in the manu- 
facturing process. Processed data support the hypothesis that the coil positioning is independent of the size of the magnet aperture. Using this hypothesis, we proved that if the reference radius is fixed at $1 / 3$ of the coil aperture, the multipoles are inversely proportional to the aperture size [see Eqs. (6) and (12)].

We used these scaling laws to derive the impact of large aperture low- $\beta$ insertions on geometric aberrations: if all of the aperture is used to house a larger beam, notwithstanding the field quality improvement geometric aberrations grow at least proportionally to the aperture [scenario 1, see Eqs. (17) and (28)]. On the other hand, if the beam size remains constant, the aberrations will rapidly decrease with a power of the inverse of the aperture size [scenario 2, see Eqs. (18) and (29)].

We showed that one can find a solution keeping the same geometric aberrations but still increasing the beta function. For instance, a double aperture in the LHC insertion quadrupole would allow in principle a higher $\beta$ function by a factor 6: this option would lead to much higher geometric aberrations, which could limit the machine performances. On the other hand, an increase of the beta function by a factor 2.5 would preserve the aberrations proportional to $b_{3}$ and to $\left(b_{3}\right)^{2}$, and would reduce the aberrations proportional to the higher order multipoles.

\section{ACKNOWLEDGMENTS}

We wish to thank L. Bottura, N. Catalan-Lasheras, P. Hagen, G. Kirby, R. Ostojic, F. Simon, S. Sanfilippo, W. Venturini-Delsolaro, the American and the Japanese teams of the LHC MQXA and MQXB, and the RHIC magnet group for providing the magnetic measurements data. We wish to acknowledge R. Gupta, L. Rossi, T. Tortschanoff, and R. Wolf for useful discussions and comments. We acknowledge the support of the European Community-Research Infrastructure Activity under the FP6 "Structuring the European Research Area" program (CARE, Contract No. RII3-CT-2003-506395).
[1] O. Bruning et al., LHC Design Report, CERN 2004-003, 2004.

[2] Y. Ajima et al., Nucl. Instrum. Methods Phys. Res., Sect. A 550, 499 (2005).

[3] R. Bossert et al., IEEE Trans. Appl. Supercond. 13, 1297 (2003).

[4] O. Bruning et al., LHC Project Report 626, 2002.

[5] T. Sen et al., in the European Particle Accelerator Conference, 2002, pp. 371-373.

[6] J. Strait et al., in the Particle Accelerator Conference, 2003, pp. 42-45.

[7] F. Ruggiero et al., European Particle Accelerator Conference, 2004, pp. 608-610.

[8] R. Ostojic et al., in the Particle Accelerator Conference, 2005, pp. 2795-2797.

[9] S. Gourlay et al., IEEE Trans. Appl. Supercond. 16, 324 (2006).

[10] R. De Maria and O. Bruning, in the European Particle Accelerator Conference, 2006, pp. 574-576.

[11] J. P. Koutchouk, LHC Project Report 973, 2006.

[12] R. De Maria, in the CARE Proceedings, Valencia, 2006.

[13] E. Todesco and J. P. Koutchouk, in the CARE Proceedings, Valencia, 2006.

[14] R. Assman, in the CARE Proceedings, Valencia, 2006.

[15] M. Anerella et al., Nucl. Instrum. Methods Phys. Res., Sect. A 499, 280 (2003).

[16] J. Herrera et al., in the Particle Accelerator Conference, 1985, pp. 3689-3691.

[17] R. Gupta, Part. Accel. 55, 129/139 (1996).

[18] W. Scandale, E. Todesco, and R. Wolf, IEEE Trans. Appl. Supercond. 10, 93 (2000).

[19] P. Ferracin, W. Scandale, E. Todesco, and R. Wolf, Phys. Rev. ST Accel. Beams 3, 122403 (2000).

[20] B. Bellesia, C. Santoni, and E. Todesco, in the Tenth European Particle Accelerator Conference, 2006.

[21] L. Rossi and E. Todesco, Phys. Rev. ST Accel. Beams 9, 102401 (2006).

[22] A. Bazzani, E. Todesco, G. Turchetti, and G. Servizi, CERN Yellow Report 94-02 (1994), Chaps. 1-2, and pp. 154-156. 